\title{
Categorizing job physical exposures using simple methods
}

\author{
Stephen Bao, Barbara Silverstein \\ ${ }^{a}$ Safety and Health Assessment and Research for Prevention (SHARP) Program, Washington State Department of \\ Labor and Industries, PO Box 44330, Olympia, WA, \\ ${ }^{\mathrm{b}}$ Deparment first, then University or Company name, Insert a complete correspondence (mailing) address, \\ Abbreviate US states, Include country
}

\begin{abstract}
Work-related musculoskeletal disorders (WMSDs) are debilitating for workers and costly for employers. Existing exposure assessment tools were modified for rapid job physical exposure and company organizational exposure assessment. These were augmented with injured worker interviews to "put the meet on the bones" in characterizing risk. These risk assessments are conducted in all industry sectors
\end{abstract}

Keywords: musculoskeletal disorders, exposure assessment, surveillance, work culture

\section{Introduction}

Work-related musculoskeletal disorders (WMSDs) are $27 \%$ of compensable workers compensation claims in Washington State, caused primarily by manual handling, repetitive motion and static loading. We hypothesize that companies which take measures to improve physical work conditions, work organization and safety culture will have fewer WMSD cases compared to those which do not consider workplace ergonomic conditions. An ergonomics surveillance project in Washington State is studying paired companies of different sizes with the highest and lowest back, shoulder, wrist and knee WMSD workers compensation claims incidence rates in each of the seven 'industry sectors with the highest overall workers compensation claims rates and numbers of WMSDs.

In order to assess physical work conditions, a set of easy to use assessment tools is needed. Selection criteria for these tools included: (1) address at least one of the WMSD risk factors, (2) relatively easy to use, (3) no need for complicated and expensive instruments, (4) able to be used among relatively large populations with minimal interruptions to the workers, and (5) able to categorize job risks. We present the development of the exposure categorization tools used in this project. WMSD injured worker interviews, work organization and safety cultures are also documented in this project.

\section{Method}

Occupational injury/illness surveillance methods require a comprehensive list of physical WMSD risk factors be created. These include high hand force, repetitive impacts, highly repetitive motion, handarm vibration, awkward postures, lifting, pushing/pulling/carrying, whole body vibration, as well as thermal comfort and lighting conditions. In order to assess these risk factors, different job evaluation tools were identified. We chose a combination of instruments to test differences between them for use in a final surveillance tool for practitioner use. These included Washington State Checklist, Strain Index, ACGIH TLV for lifting, NIOSH Lifting Equation. A two-step process is used in the job evaluation. Step 1 is a screening process which determines the existence of any potential risk factors in a job. If yes, Step 2 follows to take a closer look at these risk factors. Step 2 job evaluation tools are selected among well 
published and cited tools. The above mentioned 5 criteria are used in selecting these tools.

Another component of the WMSD surveillance project includes interviews with injured workers off work with back, shoulder, wrist and knee WMSDs. These interviews focus on perceived environmental, organizational, equipment, and task factors that may have contributed to the injury. Additionally, we collect organizational culture data from both management and worker representatives via interview, as well as injured worker interviews to better understand injury circumstances and potential solutions.

\section{Results}

The following evaluation tools were selected for the different risk factors. For the risk of high hand forces, the Washington State (WS) Hazard Zone Checklist (1), the Strain Index method (2), and the Quick Exposure Check (QEC) method $(3,4)$ were selected. The reason for using multiple methods for the same risk factor is that different methods have different levels of specificity, reliability and validity, as well as practicality. The use of multiple methods in this project will provide further information about the characteristics of these job evaluation methods which can then be used to guide practitioner selection and use of these methods.

For highly repetitive motion the WS Hazard Zone checklist (1) as well as the Strain Index (2) and QEC $(3,4)$ are used. Awkward postures are evaluated by the WS Hazard Zone checklist (1), together with the QEC $(3,4)$. Since prolonged sitting and standing are related to postures and there are no well accepted guidelines on these, duration limits of 8 hours for prolonged sitting and 6 hours for prolonged standing were used to categorize job risk levels. For manual material handling risk factors such as lifting, pushing, pulling, and carrying, the ACGIH Lifting TLV (threshold limit value) (5) and Liberty Mutual's Manual Material Handling guidelines (6) are used. Since the QEC and the WS Hazard Zone checklist also cover manual material handling risk factors, they are used to evaluate the manual material handling risk factors incorporated in this tool as well.

\section{References}

[1] Washington State Department of Labor and Industries. Hazard Zone Jobs Checklist. [Online] Available at http://www.lni.wa.gov/wisha/ergo/evaltools/HazardZoneChe cklist.PDF, 2000.
Whole body and hand-arm vibrations are evaluated according to the corresponding ISO standards (7, $8)$. Given the nature of this project, detailed instrumentation of vibration measurement is impractical, so an alternative method is used. An estimate the level of vibration was obtained by asking workers to rate their perceived vibration level on a modified Borg scale (9) when using vibration tools or driving vehicles. The published range of the type of tools/vehicles to estimate the vibration levels was used. Alternatively, the published declared vibration value of the tool was used. The actual duration of tool use or vehicle driving is obtained from workers' self-reports. Using the estimated vibration levels and reported exposure time, the 8-hour equivalent vibration levels are calculated according to the corresponding ISO standards $(7,8)$. Environmental factors ( thermal comfort, lighting) follow suggested guidelines (10). Data collection is performed using electronic tablets at the worksite..

Work culture factors were derived from management and worker interviews including turnover, safety responsibility, process improvements, light duty job availability, cooperation and conflict, feedback, taking shortcuts, reporting near misses. Common themes included good communication is an essential element of a good safety program, conflict arises when issues have been reported but not addressed, and conflict has been higher because of the sluggish economy.

\section{Discussion}

Most of the tools provide evaluation results at the job level, except the QEC which provides task level evaluations for most risk factors. Many methods have application restrictions. An application procedure needs to be developed so that these evaluation methods can be modified to be used in these complicated situations. Organizational culture provides the context for improvement possibilities in musculoskeletal risk. Organizational culture provides the context for improvement possibilities in musculoskeletal risk.

[2] Moore, JS, A Garg. The Strain Index: a proposed method to analyze jobs for risk of distal upper extremity disorders. Am Ind Hyg Assoc J. 1995;56:443-58.

[3] David, G, V Woods, G Li, P Buckle. The development of the Quick Exposure Check (QEC) for assessing exposure to risk factors for work-related musculoskeletal disorders. Appl Ergon. 2008;39:57-69. 
[4] Li, G, P Buckle. A practical method for the assessment of work-related musculoskeletal risks - Quick Exposure Check (QEC). In, Proceedings of the Human Factors and Ergonomics Society 42nd Annual Meeting, Chicago: Human Factors and Ergonomics Society, 1998: pp. 1351-5.

[5] ACGIH. Documentation of the Threshold Limit Values for Physical Agents. Cincinnati, Ohio: ACGIH, 2001.

[6] Liberty Mutual Group. Manual Materials Handling Guidelines, Boston, Massachusetts: Liberty Mutual Group, 2004

[7] ISO. Mechanical vibration -- guidelines for the measurement and the assessment of human exposure to hand-transmitted vibration. Geneva, Switzerland: International Organization for Standardization, 1986

[8] ISO. Mechanical vibration and shock -- evaluation of human exposure to whole-body vibration. Part 1 General requirements. Geneva, Switzerland: International Organization for Standardization, 1997

[9] Borg, GAV. Psychophysical bases of perceived exertion. Med Sc Spt Exer. 1982;14:377-81.

[10] Chengalur, SN, SH Rodgers, TE Bernard. Kodak's Ergonomic Design for People at Work. New Jersey: John Wiley \& Sons, Inc., 2004 